\title{
Safety of a powder-free latex allergy protocol in the operating theatre: a prospective, observational cohort study
}

\section{Allergy, Operating rooms, contamination, Anaesthesia,audit}

P. Brall ${ }^{1}$, J. Dubois ${ }^{1}$, W. Wyckmans ${ }^{1}$ L. Jamaer ${ }^{1}$, J. P. Ory ${ }^{1}$, B. Stessel ${ }^{1}$

${ }^{1}$ Department of Anesthesiology \& Intensive Care, Jessa - Hasselt (Belgium)

\section{Background and Goal of Study}

Since powdered latex gloves induce airborne latex particles with risk of airborne anaphylactic shock, the American Association of Anesthesiologists recommends that latex allergic patients must be the first undergoing treatment on the day of surgery in an operating room (OR) left unused for 3 to 6 hours. Obviously, surgery has to be postponed in the case of improper scheduling which causes patient discomfort and financial losses. The Australasian Society of Clinical Immunology and Allergy suggested that this specific scheduling is not necessary if all powdered latex gloves are removed from the $O R$. Since there is currently no evidence confirming the safety of this practice in a clinical setting, we investigated if latex allergic patients could be treated safely without special scheduling requirements when all powdered latex gloves were removed from the OR.

\section{Materials and Methods}

We first removed all powered gloves from the OR in our hospital and informed all involved healthcare workers about the new protocol. Afterwards we changed our policy for scheduling latex allergic patients, which from now on could be scheduled throughout the day without special requirements.

All patients with (a high risk for) latex allergy scheduled for surgery in our hospital were prospectively enrolled from 10/2015 to $11 / 2016$. A baseline questionnaire prior to surgery assessed the patient's history of latex allergy. Any allergic perioperative reactions were recorded by the anesthesiologist. Delayed or late-phase allergic reactions were assessed by telephone call 72 hours postsurgery.

\section{Results and Discussion}

In total, 229 patients were enrolled. No patients showed a type 1 allergic reaction. Only 1 patient suffered from an irritant contact dermatitis directly after surgery. Eight patients (3.5\%) reported a type IV latex allergic reaction $72 \mathrm{~h}$ post-surgery, of which $3(1.3 \%)$ reported local pruritis, $4(1.7 \%)$ reported local rash and $1(0.4 \%)$ reported local rash with blisters. General symptoms included cough $(n=1(0.4 \%))$, asthma $(n=113 / 12 / 20172 / 2$ $(0.4 \%))$, problems with the eyes $(n=1 \quad(0.4 \%))$, gastro-intestinal problems $(\mathrm{n}=6 \quad(2.6 \%))$, headache $(n=2(0.9 \%))$ and general pruritis $(n=2$ $(0.9 \%))$.

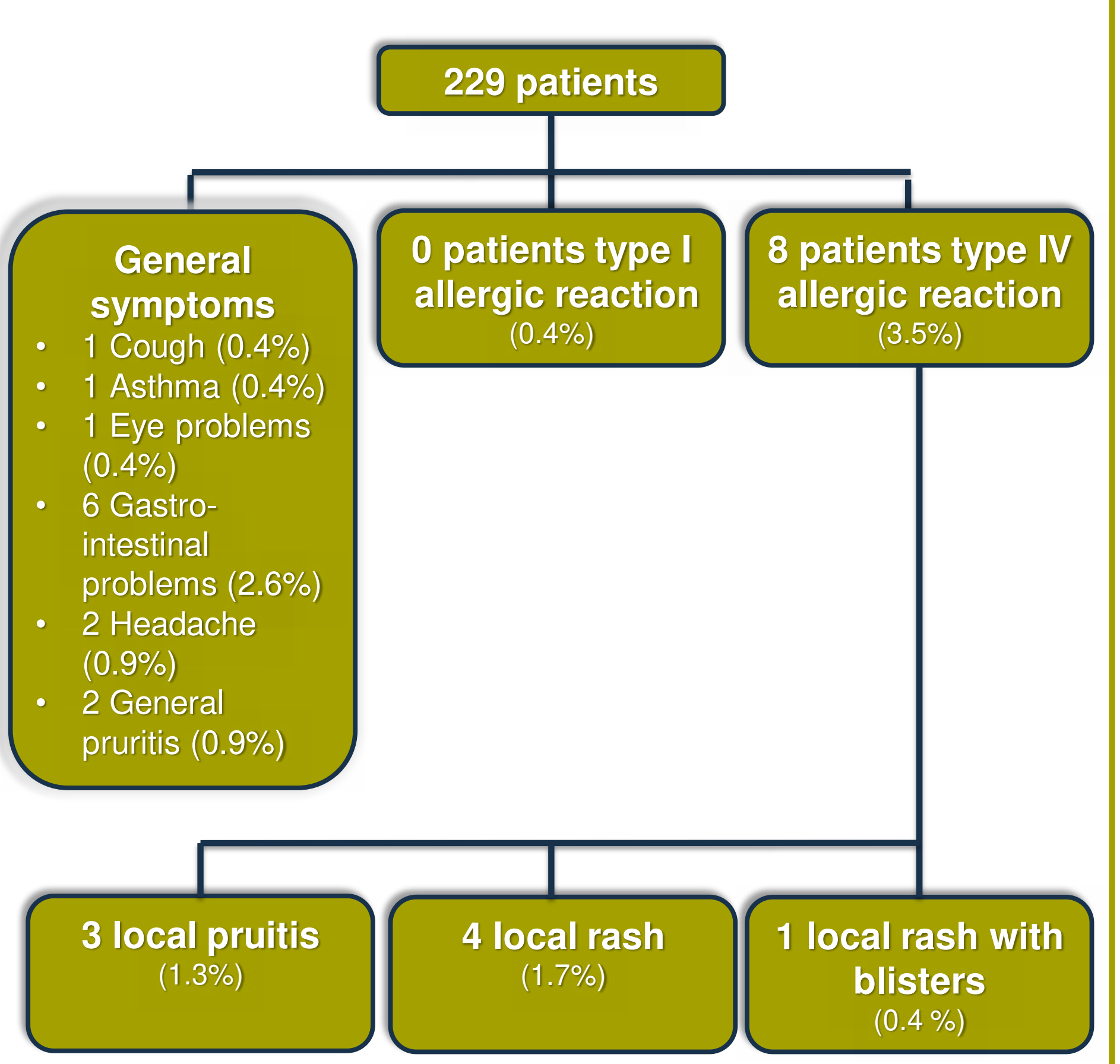

\section{Conclusion(s)}

The new protocol, that allows scheduling latex allergic patients throughout the day in an OR that is totally free of powdered latex gloves is considered safe in our pilot study. However, large epidemiologic trials are necessary to confirm this findings. 